\title{
INTEGRATED KNOWLEDGE ON A THREE-DIMENSIONAL BASIS: THE FOUNDATION OF THE CONSERVATION PROJECT AND POSSIBLE IMPLICATIONS IN THE BIM AREA THE CASE OF THE PORTRAIT ROOM OF PALAZZO BOREA D'OLMO IN SANREMO
}

\author{
C. Campanella ${ }^{1}$, S. Alauria ${ }^{2}$, L. Amatori ${ }^{3} *$ \\ ${ }^{1}$ Politecnico di Milano, Italy - Dep. ABC - christian.campanella@ polimi.it \\ ${ }^{2}$ Freelance architect - info@3deffe.com \\ ${ }^{3}$ Freelance engineer - info@studioamatori.com
}

Commission VI, WG VI/4

KEY WORDS: design, surveying, photogrammetry, laser scanner, restoration, conservation, project, BIM

\begin{abstract}
:
This involved a sort of preventive diagnostics based on the historical knowledge of the building, on the construction techniques and on the compositional style, on the other hand on diagnostic imaging using photographic techniques developed from the early 1800 s. The preliminary cognitive approach, the stylistic identification, the classification of method, the historical reading, the critical analysis of the historical and compositional genesis of the building, the careful reading of the pathogenesis in progress, mark, from the mid-800 in then, the evolution of the concept of restoration that will take shape from time to time from what was "before" and only secondarily from what "is now". This perhaps unconscious diagnostic reading of the artifact (first artistic, then monumental and finally as a cultural testimony) has, in fact, involved generations of restorers painting the landscape of the restoration sometimes in different way, with results that well we know.

In fact, almost never the fathers of restoration (or anti-restoration) explicitly touch the issues of preventive diagnosis, but certainly base their theories and their "restoration" on the observation of the factory, on the knowledge of construction techniques and historical period of belonging.
\end{abstract}

The things begin to change starting from the first Charter of Restoration of 1883 with the enlargement of the diagnostic project (which finds paternity starting from the 1972 Italian Charter, which embraces many disciplines: from photography to relief, from chemistry to physics, from the historical reading to the knowledge of the materials, from the identification of the material pathogenesis to the reading of the static and structural framework of the buildings.

With the passing of time and the explosion of science and technology in the field of cultural heritage all aspects related to preliminary knowledge aimed at their conservation evolve exponentially especially with the advent of electronics. Starting from the 60 s of the 1900s, equipment with great calculation capacity and small dimensions was born, a real springboard for the new techniques of surveying by means of a laser scanner, photographic socket and photogrammetric restitution.

Tools and techniques of restitution settle and even more solidly base the knowledge base linked to the project of conservation and enhancement of cultural heritage.

Base of support and extraction of selected data and / or dedicated to the project continuously interface with the disciplines of knowledge that are now more and more "forced" to the table of confrontation to reveal information hidden in the folds of time.

A recent work on an eighteenth-century Sanremese building it gives us information on the complexity of the construction of a process of knowledge articulated, composed of several activities to be correlated and integrated continuously, one in the other to try to give answers on evident problems of degradation never fully documented.

The study, performed in a rather limited time frame, focused on the Hall of family portraits seriously degraded by "accidents of various kinds". The work was based on the three-dimensional conception of the acquired data, allowing to investigate the hall as an articulated organism, supporting the technicians in the three-dimensional understanding of the asset, constituting a complete database of the actual state, becoming support of the results of the various surveys conducted for the knowledge of the asset.

In this cognitive path, the BIM method is understood as the possibility of constructing the "as built" model, complete not only of the dimensional graphic data of the asset, but also of those that contribute to the determination of the actual state. The graphic aspect of the model must be connected with historical, material, degradation, contextual information, with the results of any specialized investigations conducted.

Only then will the BIM model of a historic building be the complete database, the "medical record" of its state of health, complete with diagnosis, care, indications of maintenance that can be shared and questioned over time.

\footnotetext{
* Corresponding author
} 


\section{INTRODUCTION}

\subsection{The diagnosis in the architectural restoration}

The concept of diagnosis in the field of architectural "restoration" has always been based on the need, built over the centuries, to know the patient before developing the intervention therapy.

References to knowledge, medicine and treatments to be provided through a purely qualitative and descriptive analysis, since the first studies provided by Vitruvio (De architectura, Book I, 15th c.), has undergone an increasingly marked scientific and methodological characterization. One of the first clear references comes from Leon Battista Alberti pointing his finger at the defects of the buildings (De re aedificatoria, book $X$, chapter I, 1450), opening a search line that will lead us to the end of the nineteenth century. This will be followed by the Filarete (Treaty of Architecture, Book I, f.61, 1464), Palladio, Scamozzi and many others in dealing with a problem that was not substantially related to the care of the patient, its recovery, the preservation of matter degraded by adopting sophisticated and conservative techniques, but to correct the defects of the monument object of intervention.

This involved a sort of preventive diagnostics based on the historical knowledge of the building, on the construction techniques and on the compositional style, on the other hand on diagnostic imaging using photographic techniques developed from the early 1800 s.

\subsection{The knowledge as basis for conservation and enhancement}

Knowledge is the fundamental and inalienable requirement of any conservative and innovative design that the design specialist can move towards a cultural asset.

Knowledge is awareness, is responsibility, is control.

Knowledge is the guideline. It is an instrument of participation of an architecture; it is an intimate way of communication with it. It is a humble and patient process of research and investigation.

The Knowledge is got through the actions of investigation of an object in all its parts. The practical outcome of the cognitive path is the awareness of the design action: it is the database of information that, in every aspect of the intervention, leads to the conservation and enhancement of the good itself, respecting its nature as "material testimony having the value of civilization".

Only the complete and accurate knowledge of the building can lead to choices scientifically and culturally compatible with its historical authenticity and architectural individuality.

Each cognitive journey is a new adventure because every building is different in terms of history, location, exposure, materials, technical details, transformations.

The architect must be able to listen, feel and identify these peculiarities and, based on these, undertake his adventure free from preconceptions.

As a "doctor", the architect, only after having listened to his patient, identifies and implements the most suitable investigation methods in preparation for recognizing the outward manifestations of the illness, identifying the causes and formulating the diagnosis.

\section{THE CASE OF THE PORTRAIT ROOM OF PALAZZO BOREA D'OLMO IN SANREMO}

\subsection{Introduction: objectives and method of investigation}

The case study here presented is the eighteenth-century Palazzo Borea d'Olmo, in Sanremo. On the Palace, a cause of its historical, artistic, architectural peculiarities, and its involvement in the history of the City, are a protection and an environmental restriction, according to Law 1089/1939 and Law 42/2004. The recent study, conducted over a fairly limited period of time, focused on the Family Portrait Room on the second noble floor of the Palace.

The Hall is decorated with eighteenth-century frescoes and it has been seriously damaged by "accidents of various kinds", strengthened over the time and manifested themselves in a repeated manner, never fully fronted and solved.

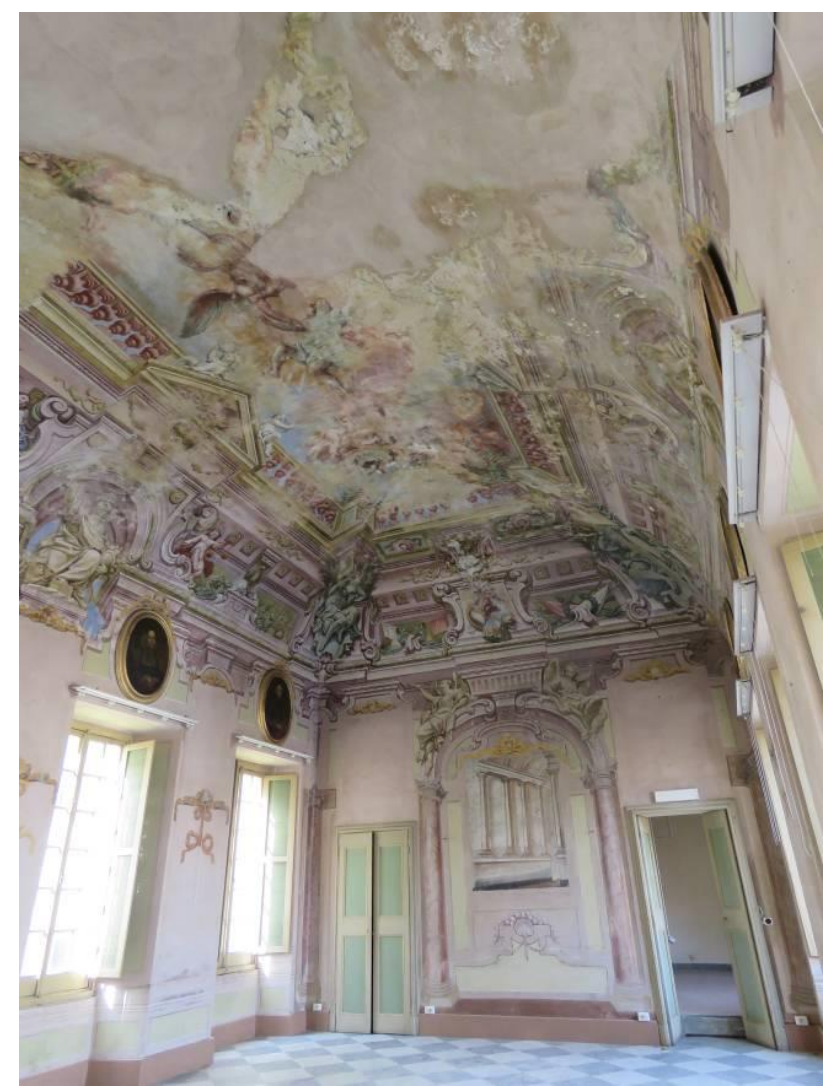

Figure 1. Family Portrait Room on the second noble floor of the Palace Borea d'Olmo, Sanremo (IM).

The research had the following double objective:

1. identify and differentiate the causes of degradation;

2. identify the most probable period of manifestation of the forms of degradation in progress

The lack of historical, graphic and photographic documentation, the lack of proofs related to the historical transformations, even traumatic ones, suffered by the artefact over the time; the lack of information on the use of the building (residential before, museum after) and the lack on the maintenance of the hall: all these elements have made essential to face a cognitive path "from scratch" where the artefact was therefore approached as the focus of attention and the main source of information.

The study was based on the three-dimensional conception of the acquired data: architecture was investigated as an articulated 
"living" organism; the technical team moved towards its organic understanding, gradually completing and strengthening a model conceived as a multidisciplinary database of the current state of the room.

In this context, the geomatic and three-dimensional survey methods, with laser scanners and digital photogrammetry, in the case in question, have:

1. helped in the planovolumetric understanding of the building;

2. made possible the building of a navigable 3D model;

3. made possible the representation of the results of the investigations, with effective and integrated graphics

The three-dimensional survey is, therefore, a powerful instrument to:

1. research,

2. interdisciplinary knowledge,

3. representation.

In the path of research, the BIM method is an important resource. In particular, the "as built" model of the building, includes not only geometric information, but also descriptions and details useful for the characterization of the actual state of the building.

The BIM model of the protected building includes information about the history, the materials, the progress of the degradation, the results of specialist study.

Only in this way the BIM model of the historic building will be complete. Effectively it will be the "medical record", the photography of this health. $\mathrm{x}$

\subsection{Performed analyses}

The starting point of the building analysis has been the Family Portrait Room itself, in particular because of the absence of documentation about its history.

The first step: the observation of the site and the signs of the history. During this initial phase, the role of the architect has been passive, and the building has revealed itself like a sick person in front of his doctor.

Afterwards the observation of the symptoms, the doctor has carried on with the next "active" phase of survey:

4. Bibliographic, archive and document analysis;

5. non-destructive instrumental investigations:

- 3D Survey with laser scanner;

- photogrammetric survey of the frescoed vaults;

- thermographic survey

- close-up analysis to the vault.

The integrated study method, (laser scanner, photogrammetry, thermography and non-destructive instrumental investigations) has allowed the achievement of the diagnosis about the health of the Family Portrait Room.

The team of specialists, in this phase, intentionally carried out only the non-destructive investigations.

If we had chosen to operate according to invasive methods, the choice of points where to make samples would be based on a selection conducted only on personal criteria. It would not have been possible, therefore, to determine, without a preliminary general analysis, the real representativeness of the samples with respect to the organism.

The continuous feedback between the survey phases and the results of the investigations has led, step by step, to obtain the level of knowledge of the building necessary to reconstruct its history, identify the causes of degradation and the most probable period of their manifestation. The starting point of all of the data analysis has always been the 3D model that has been explored in RGB, DEM, reflectance, mesh and point cloud.

\subsection{Results of analysis}

\subsubsection{Bibliographic, archive and document analysis.}

They allowed to identify the main historical phases of the life of the building identifying over time the moments and the causes of the transformations.

The most ancient testimonies date back the original nucleus of the Palace to the beginning of the sixteenth century. However, the historical bibliography about the construction phases, that led to the current architectural and artistic conformation, are really limited.

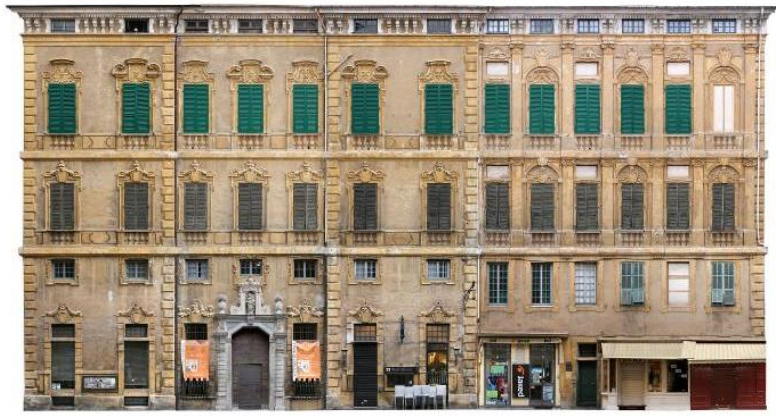

Figure 2. Main front of the Palazzo Borea d'Olmo, Sanremo (IM), Via Matteotti.

The building is located in the historic center of Sanremo. It has five floors above ground, a basement and an attic mezzanine floor (today, its current function is residential).

The structure of entire building is stone masonry with big stone bearing walls and thinner brick structural walls; the vaults of the second noble floor, (the one where the Family Portrait Room is located) are made by masonry structure.

The roof structure is in pitched wood with tile covering. Only the northern portion, above the Family Portrait Room, has been replaced by a terrace. The time of realization of this modification is unknown, but from the observation of the historical photographic documentation it is ascribable to the period between 1860 and the immediate second post-war period. The pictures below, n. 3 and 4 , show the Palace in the 1860 and 1950 year.

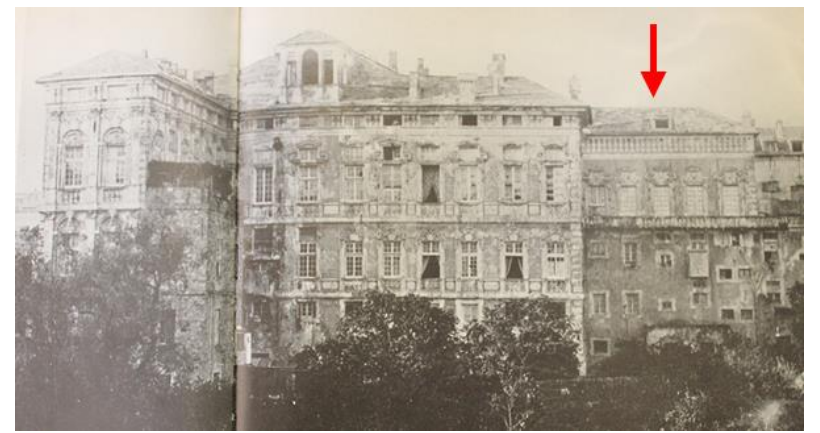

Figure 3. collection Guido Orazio Borea d'Olmo, iconographic text "Sanremo com'era", Editrice Collins, Milano 1974. 


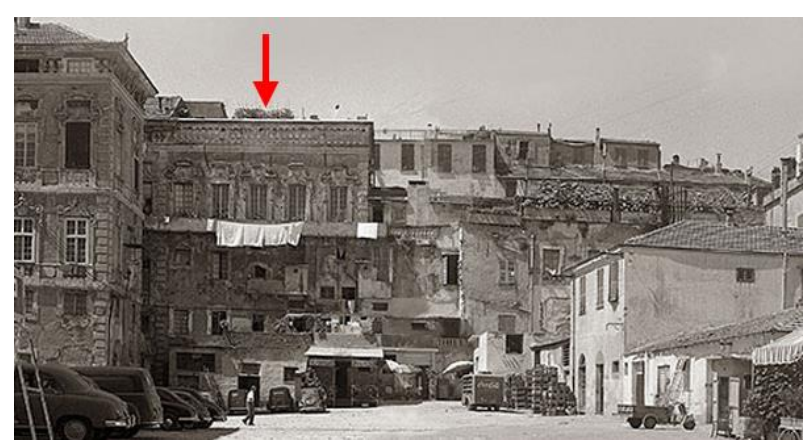

Figure 4. Photo archive Moreschi, Sanremo. Piazza Borea, 1950.

The reasons of the modification are still unknown, however, in the historical period indicated, the city of Sanremo heavily suffered the traumatic effects of the earthquake in February 1887 (known as the "Bussana Earthquake") and of war bombings in 1944.

The historical bibliography confirms that these events had destructive consequences on the urban fabric: in particular the bombings of 1944 razed to the ground the nearby Piazza Colombo and caused huge collapses even in the buildings facing the homonymous Piazza Borea.

Today, the observation of the Family Portrait Room allows us to read two other important transformations:

1. The persistence, on the façade, of the bolted end-plate of metal chains that have been cut and removed inside the building.

2. The reconstruction of a large portion of the vault, recognizable by the different building technique and by the loss of the fresco decorations.

The historical analysis has allowed to bring back the reasosns of such transformations to the historical traumatic events that several times involved the buildings of the historical center of the City between the end of 1800 and the second half of 1900 .

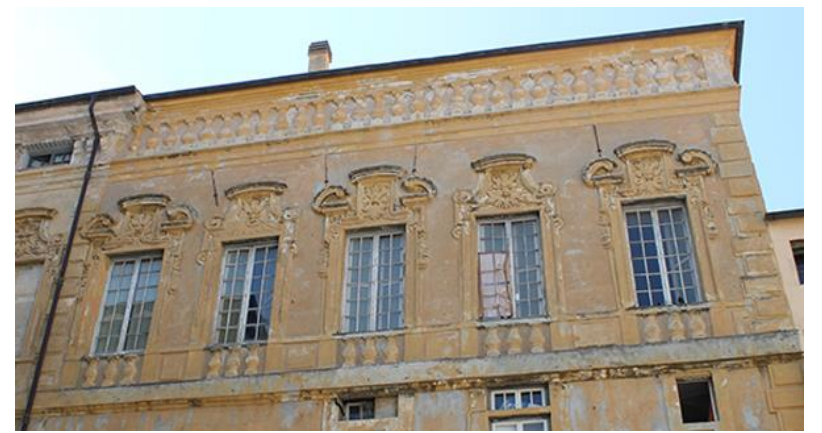

Figure 5. External view of the Family Portrait Room, 2018

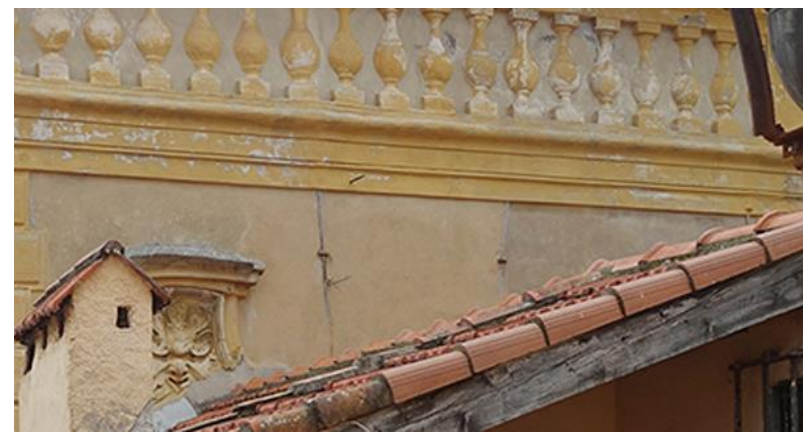

Figure 6. External view of the Family Portrait Room, 2018

In general, the historical and bibliographic photographic images, representing the exteriors of the Palace from the second half of the 1800 s to the second post-war period, point out a decadent state of preservation.

The archival sources give news of a single restoration of the facades, which given the extension and the articulation of the building, was realized in several stages, between 1974 and 1985 , involving the different parts of the facades step by step.

No maintenance and restoration work, on the other hand, is documented on the terrace above the Portrait Room except for the urgently constructed one in 1994: the serious and extensive degradation that affected the flooring, the balustrades, the rainwater disposal systems, in fact caused infiltrations and damage to the underlying frescoed vault. This extensive degradation was also damaging the underlying frescoed vault, with infiltration and humidity. Communications and letters between the Palace Property and the Authority denounced the deterioration and urgently required a restoration intervention.

So, the historical analysis and the archival research have allowed us to circumscribe in the time the architectural and structural transformations of the Hall of Portraits but they also confirmed the lack of data concerning both the condition of the frescoes and the restoration and conservation measures.

\subsubsection{Integrated laser scanning and photogrammetry survey:}

It allowed to reproduce in a highly detailed photorealistic way the state of conservation of the frescoed surfaces. The 3D object scanning helps to improve the design process, accelerates and reduces data collection errors, saves time and money making it an attractive alternative to traditional data collection techniques. 3D scanning is today successfully used for mapping historic buildings.

With digital photogrammetry, instead, it has been possible to create a texturized model of the frescoed works, faithful to reality under the chromatic profile. The final model, point cloud, mesh and texture, allowed the deep analysis of the surfaces in the photorealistic, geometric and degradation aspects.

The possibility of displaying the point cloud in false colours allowed us to detect the differences in materials according to their behaviour in light (reflectance, absorbance, light transmittance) and in relation to their distance from the gripping point (DEM processing, Digital Elevation Model):

- The RGB visualization provides realistic colour photographic image (Fig. 7);

- The DEM visualization can be set with customizable colour scales in order to obtain the best graphic data reading. The DEM represents the distribution of heights in relation to the base level 0.00 (level of first scan). The DEM therefore highlights the deformations, the lesions and the discontinuities of the vault due to the different reconstructions. It emphasizes the swellings and plaster detachments attributable to degradation, infiltration, prolonged action and repeated humidity (Fig. 8);

- The reflectance visualization scale (Fig. 9), customizable in order to obtain the best reading of the data, highlights the distribution materials according to their light reflection level. In this way it is possible to distinguish the materials with lower reflectance (greater absorption of the laser beam) and those with higer reflectance (low absorption of the laser beam). 


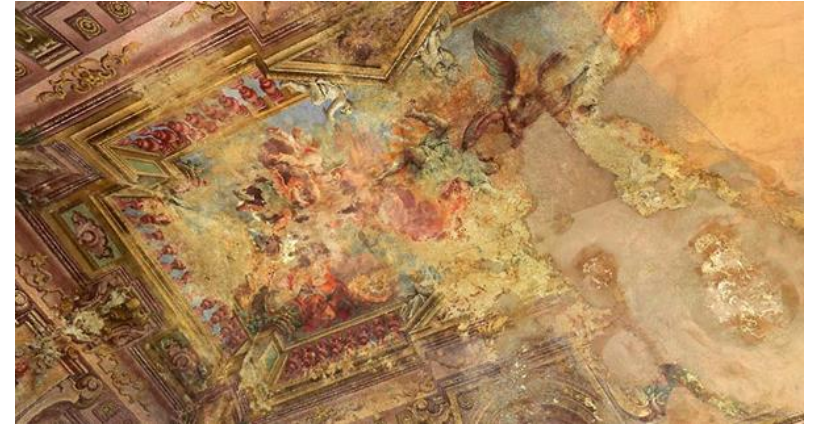

Figure 7. RGB three-dimensional view - 3d model of the vault elaborated with photogrammetry.

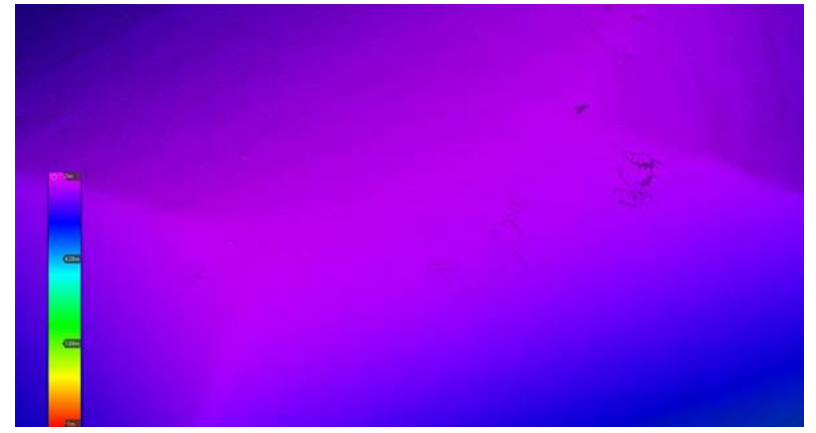

Figure 8. DEM three-dimensional view - 3d model of the vault elaborated with laser scanning

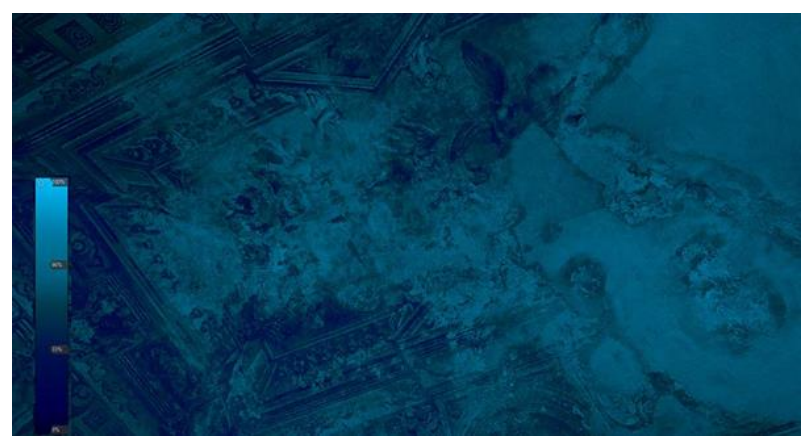

Figure 9. visualization in reflectance scale (custom colours) - 3d model of the vault elaborated with laser scanning

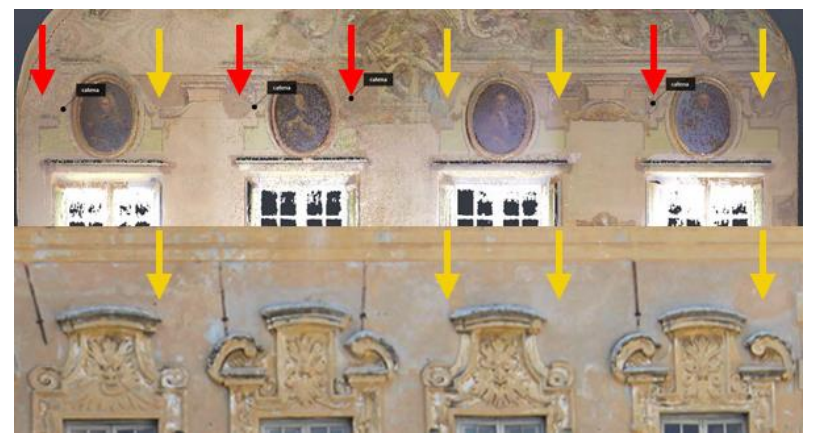

Figura 10. Interior and exterior view of the Family Portrait Room. The red arrows indicate the existing bolted end-plate; the yellow arrows indicate the plaster swellings detected where the bolted end-plate are no longer in place.

The survey methodology allowed us to highlight the structural discontinuities, the plaster reconstruction areas, the zones most affected by infiltration and humidity.
The result has been particularly effective to find out the plaster resumptions where the metal chains were removed even if today the external metal end-plate no longer exist (Fig. 10).

In these points, the layers of plaster and paint have marked swellings and detachments that can be seen with the DEM display of the point cloud. The comparison with its RGB version allowed us to verify the chromatic difference of the plaster. (rif. Fig. 10, 11, 12).

The comparison, instead, between the RGB view of the 3D model (dense cloud) and the reflectance view has clearly highlighted the problems of humidity and infiltration in the contact areas between the original masonry vault and its rebuilt portion after the collapse.

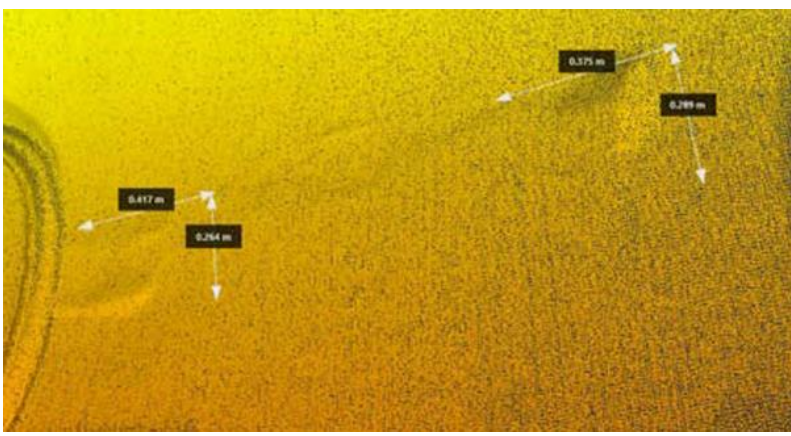

Figura 11. DEM three-dimensional view of the dense cloud: detail of the points where the metal chains have been removed.

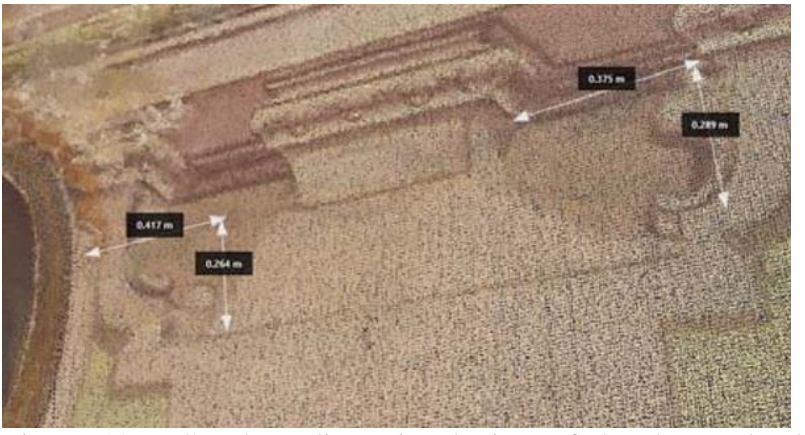

Figura 12. RGB three-dimensional view of the dense cloud: detail of the chromatic differences of the plaster in the points where the chains have been removed.

\subsubsection{The infrared thermography.}

It is the non-invasive investigation technique, applied to the theory of building surface pathologies, which exploits the physical principle according to which any other system is in the form of infrared radiation. The instrument that converts the infrared energy emitted by a body into a digital signal is the infrared camera. This tool allows you to view in radiometric infrared (thermogram): the thermal imager acquires the distribution of temperatures in an object and exploiting algorithms returns a thermal image of a distribution in false colours.

The infrared camera captures the temperature allocation of a surface, without need of contact with it: this allocation can be always viewed as visible information. The temperature can also be measured in real time.

In our case study, the thermographic survey was carried out in February at 11 a.m. The day was sunny, the average outdoor temperature was around 11 degrees.

The thermographic analysis did not show any active moisture phenomena due to infiltration. 
As can be seen in the image below (fig. 13), the temperatures detected at the lower level of the frescoed vault were very close to the external temperatures.

The causes are attributable to:

- Long-time lack of environmental air conditioning system at the time of the survey;

- degradation of walls, window parapets and sills;

- presence of lesions on the vault and on the perimeter walls;

- degradation of the windows wooden frames, lack and breakage of some panes,

- general humidity in all rooms of the second noble floor;

- presence of several infiltrations (and therefore of persistent humidity) in the other rooms.

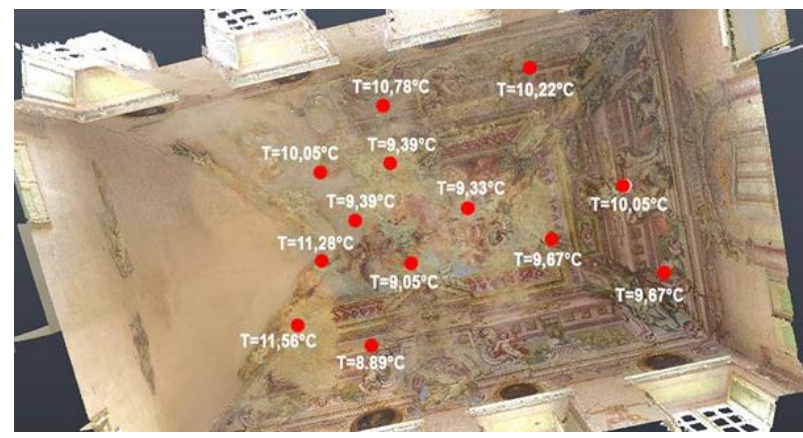

Figura 13. Temperature distribution to the intrados of the vault detected with a thermal imaging camera.

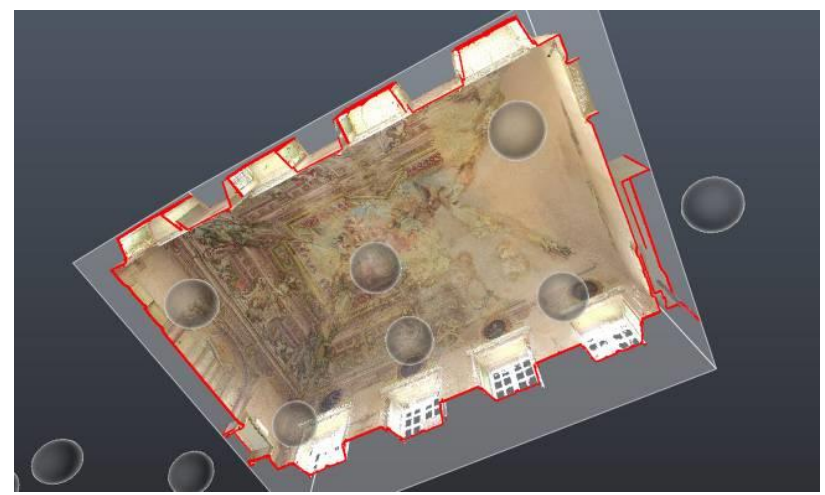

Figura 14. RGB three-dimensional view - 3d model of the vault elaborated with laser scanner

The result of the thermographic survey confirmed that the plaster and frescoed surfaces are continuously and cyclically subject to sudden changes in temperature and humidity.

The deficiencies observed at the structural level and the already highlighted reasons are the main cause of the heavy degradation of the frescoes (rif. Fig. 11).

\subsubsection{The investigations by sound typing and auscultation.}

They were carried out with close macroscopic observation of the vaulted surface of the Family Portraits Room.

The objective was to compare, with a further non-destructive specialist analysis, the results of the previous instrumental investigations and to deepen the knowledge of the current degradation: entity, causes and period of manifestation.

Close investigations showed the following forms of degradation:

- detachment between the plaster layers, deformations and swelling especially in the areas affected by previous interventions (points where the metal chains were removed, areas of contact between the original vault and the portion redone after the collapse);
- presence of atmospheric particulate on the entire surface of the vault;

- salt efflorescence, patinas and whitish concretions with particular concentration in correspondence with the reintegrations;

and the corresponding causes:

infiltrations, no longer active but currently confirmed and proved by archival documentation from the early 1990s;

absence of environmental humidity control both due to the lack of a thermo-hygrometric control system and to the degradation of windows and walls

- the detachment between the walls.

In conclusion, this additional specialist analysis, conducted directly on the frescoes, has confirmed the results of previous historical research and non-destructive investigations (laser scanner, photogrammetry and thermography).

\section{CONCLUSIONS}

Today, the infiltration and humidity damage on the vault of the Family Portraits Room affects almost the entire surface. The damages, confirmed by analyses conducted with different non-invasive techniques, can be attributed to several causes. These causes occurred over the time, starting in the late 1800s. They are due to traumatic events and to repeated humidity phenomena. The historical analysis has shown that the traumatic events probably happened between the end of the nineteenth century and the first half of the twentieth century and involved the transformation of the wooden roof into a terrace. Noninvasive investigations have confirmed the presence of cracks, structural and architectural deficiencies and environmental degradation. These deficiencies are identified as the main cause of repeated and cyclical degradation, triggered at least since the 1990s. Proof of this is that the investigations by sound typing and auscultation have underlined the execution, in unspecified period, of restoration and safety interventions of the frescoes.

The archival sources do not report on this, but various phases of intervention on the pictorial works are clearly visible, carried out with the aim of recovering the frescoes and stopping the degradation in progress. In addition, a general poorly executed restoration care was observed. This may have been voluntary (distinction of the intervention with respect to the original fresco) or necessary (low financial resources).

The multidisciplinary investigations have confirmed that:

- about $30 \%$ of the frescoed vault was rebuilt at least 70 years ago,

- a serious damage from infiltration and humidity was confirmed in 1994, justifying the urgent execution of works to rebuild the terrace,

- after the reconstruction of the 1994 terrace, the restoration of frescoes, facades, masonry, parapets and windows were not documented,

- current degradation is exactly as documented in photographic publications dating back to the year 2000,

- general deterioration of the building and lack of climate control system has increased the frescoes deterioration over the last 20 years

In conclusion, the causes of degradation linked to the collapse in the period 1870-1955 are many: pre-reconstruction infiltrations in 1994, infiltration by masonry, fixtures, lack of maintenance and non-existent environmental control.

The work lead on by the team of specialists with different professional skills has produced the desired answers that have been all collected in a single model. The outcome is now easily accessible, editable and implementable on a BIM basis for the future maintenance and restoration of the building. 